\title{
ASYMPTOTIC STABILITY OF NAVIER-STOKES FLOW PAST AN OBSTACLE
}

\author{
PIOTR BILER \\ Instytut Matematyczny, Uniwersytet Wrocławski \\ Pl. Grunwaldzki 2/4, 50-384 Wrocław, Poland; \\ Institute of Mathematics, Polish Academy of Sciences, Warszawa (2002-2003) \\ E-mail: biler@math.uni.wroc.pl \\ MARCO CANNONE \\ Laboratoire d'Analyse et de Mathématiques Appliquées \\ Université de Marne-la-Vallée \\ Cité Descartes-5, bd Descartes, Champs-sur-Marne \\ 77454 Marne-la-Vallée, Cedex 2, France \\ E-mail: cannone@math.univ-mlv.fr \\ GRZEGORZ KARCH \\ Instytut Matematyczny, Uniwersytet Wrocławski \\ Pl. Grunwaldzki 2/4, 50-384 Wrocław, Poland; \\ Institute of Mathematics, Polish Academy of Sciences, Warszawa (2002-2003) \\ E-mail: karch@math.uni.wroc.pl
}

\begin{abstract}
Results on the asymptotic stability of solutions of the exterior Navier-Stokes problem in $\mathbb{R}^{3}$ are proved in the framework of weak $L^{p}$ spaces.
\end{abstract}

1. Introduction. Our aim in this paper is to prove an asymptotic stability result for small solutions of the exterior problem for the Navier-Stokes equations

$$
\begin{aligned}
u_{t}-\Delta u+(u \cdot \nabla) u+\nabla p & =f, \quad x \in \Omega, t>0, \\
u(x, t) & =0, \quad x \in \partial \Omega, t>0, \\
\nabla \cdot u & =0, \quad x \in \Omega, t>0,
\end{aligned}
$$

2000 Mathematics Subject Classification: Primary 35Q30; Secondary 76D05, 35B40.

Key words and phrases: incompressible Navier-Stokes system; exterior domain; asymptotic stability of solutions.

The paper is in final form and no version of it will be published elsewhere. 


$$
\begin{aligned}
u(x, 0) & =u_{0}(x), \quad x \in \Omega, \\
\lim _{|x| \rightarrow \infty} u(x, t) & =u_{\infty}, \quad t>0 .
\end{aligned}
$$

Here $\Omega \subset \mathbb{R}^{3}$ is an exterior domain, i.e. the complement of a compact set $K$ with a smooth boundary $\partial \Omega$. The initial condition $u_{0}$ is supposed to be divergence free in the sense of distributions, and it is attained in the weak sense as $t \searrow 0$. The condition at infinity (1.5) is understood in the sense of the weak Lebesgue space (or the Marcinkiewicz space) $L_{\sigma}^{3, \infty}(\Omega)$ of divergence free distributions belonging to $L^{3, \infty}(\Omega)$. Following the physical interpretation of the above problem, the set $K$ represents an obstacle, and the Navier-Stokes flow has the asymptotic velocity at $|x|=\infty$ equal to $u_{\infty}$. In particular, if $u_{\infty}=0$, the flow is at rest at infinity.

There is a huge amount of publications analyzing solutions to the exterior problem (1.1)-(1.5); here, we mention only some of them where the interested reader can find more complete references.

Leray [14] studied the stationary problem associated with (1.1)-(1.5) and proved the existence of a smooth steady state with a finite Dirichlet integral. Later, Finn [6] gave an asymptotic analysis for the so-called "physically reasonable" solutions proving a uniqueness result for small data in such a class. Moreover, his solutions present as well a paraboloidal wake structure behind the obstacle. We refer the reader to the two volume monograph by Galdi [7] for several other results on the stationary exterior problem and for the most complete bibliography. Here, we also quote the recent paper by Shibata and Yamazaki [19] on the behavior as $|x| \rightarrow \infty$ of stationary solutions to the exterior problem.

In the case of nonstationary solutions, Heywood proved [9] that for small data one can solve the, so-called, stability problem for physically reasonable solutions. In other words, for small data one can solve the equations obtained by replacing in (1.1)-(1.5) with a perturbed flow, with respect to smooth $L^{2}$ perturbations, within the class of physically reasonable solutions. As far as the behavior as $t \rightarrow \infty$ of nonstationary solutions is concerned, this result was later improved by several authors, (see [1, 2, 3, 5, 8, 9, 13, 17, $18,20]$ for further and more detailed accounts on the subject). However, Finn pointed out in [4] that if $u(x)$ is a stationary physically reasonable solutions and if the external force exterted on the obstacle does not vanish, then $u(x)-u_{\infty}$ is not square integrable. This is why it appeared interesting to study the existence of solutions to (1.1)-(1.5) within a class of functions larger than $L^{2}(\Omega)$.

In this direction the most comprehensive theory, that applies in a more general framework that the energy one, was that making a systematic use of $L^{p}-L^{q}$ estimates of the Stokes semigroup. These techniques were applied in the context of various function spaces and related problems (cf. e.g. $[2,3,8,10,13,18,20]$ ). Compared to the Navier-Stokes problem in the whole space $\mathbb{R}^{3}$, the case of an exterior domain is more difficult because the scaling properties of the equations are harder to exploit.

One of the most important problems is to find an adequate functional setting. There have been many approaches to the exterior Navier-Stokes problem, including Lebesgue spaces, anisotropically weighted Sobolev spaces, Morrey-Campanato spaces. Here, our choice is that of weak Lebesgue spaces $L^{p, \infty}(\Omega)$ that contain functions with the decay for 
$|x| \rightarrow \infty$ expected for the solution from physical similarity arguments. However, to get suitable estimates we need to use also a larger scale of Lorentz spaces $L^{p, r}(\Omega)$.

In this paper, we prove a result on the asymptotic stability of solutions for (1.1)-(1.5) whose existence has been recently proved by H. Kozono, Y. Shibata and M. Yamazaki in $[12,13,17,18,19,20]$; see also the work [3] of N. Depauw. The plan of the paper is the following. First, we gather the information on the linearized evolution problem, i.e. on the Stokes and Oseen semigroups. Then, we recall the results on the existence and stability of solutions. Our main results on the asymptotic stability of solutions are contained in Theorem 3.4 (the case $u_{\infty}=0$ ) and in Theorem 3.5 (the general case with $u_{\infty} \neq 0$ small enough). Finally, we will analyze the regularity of solutions in Theorems 4.1 and 4.2.

Notation. Here $L^{p, r}$ denote the $L^{p, r}(\Omega)$ Lorentz spaces of vector functions with values in $\mathbb{R}^{3}$, and $L_{\sigma}^{p, r}(\Omega)$ - their respective intersections with the divergence free (in the distributional sense) vector fields. The case $r=\infty$ corresponds to the Marcinkiewicz (or weak Lebesgue) spaces. The letter $C$ denotes inessential constants which may vary from line to line, but do not depend on particular solutions.

2. Stokes and Oseen semigroups. Since we will use the Duhamel type formulas for representing the solutions of the full nonlinear problem, estimates on the linearized problems are of importance.

Given $u_{\infty} \in \mathbb{R}^{3}$ we consider the linear problem

$$
\begin{aligned}
v_{t}-\Delta v+\left(u_{\infty} \cdot \nabla\right) v+\nabla q & =0, \quad x \in \Omega, t>0 \\
v(x, t) & =0, \quad x \in \partial \Omega, t>0, \\
\nabla \cdot v & =0, \quad x \in \Omega, t>0, \\
v(x, 0) & =v_{0}(x), \quad x \in \Omega,
\end{aligned}
$$

called the Stokes problem for $u_{\infty}=0$, and the Oseen problem if $u_{\infty} \neq 0$. The corresponding semigroups are called the Stokes and the Oseen semigroups, respectively, and are denoted by $T(t)$ and $T_{u_{\infty}}(t), t \geq 0$.

The semigroups $T(t)$ and $T_{u_{\infty}}(t)$ have been studied for (at least) 75 years, cf. [16]. Here, we recall only the contribution made by Shibata and Yamazaki in $[17,18,20]$ which is important for the topic studied in the present paper.

The main technical tools used in the present paper are the Lorentz space estimates of the Stokes and Oseen semigroups which are consequences of usual Lebesgue spaces $L^{p}-L^{q}$ estimates, cf. [11], via an interpolation argument.

Thus, we begin with the estimates for the Stokes and Oseen semigroups.

Proposition 2.1. Assume that $1<p \leq q<\infty$ and $v_{0} \in L_{\sigma}^{p}(\Omega)$. There exists $\varepsilon_{0}>0$ such that for all $u_{\infty} \in \mathbb{R}^{3}$ such that $\left|u_{\infty}\right| \leq \varepsilon_{0}$ we have

$$
\left\|T_{u_{\infty}}(t) v_{0}\right\|_{L^{q}} \leq C t^{-\frac{3}{2}\left(\frac{1}{p}-\frac{1}{q}\right)}\left\|v_{0}\right\|_{L^{p}}
$$

for all $t>0$ (here, $T_{u_{\infty}}(t)=T(t)$ for $\left.u_{\infty}=0\right)$. Moreover, the gradient of the semigroup satisfies the estimate 


$$
\left\|\nabla T_{u_{\infty}}(t) v_{0}\right\|_{L^{q}} \leq c t^{-\frac{3}{2}\left(\frac{1}{p}-\frac{1}{q}\right)-\frac{1}{2}}\left\|v_{0}\right\|_{L^{p}}
$$

for $1<p \leq q \leq 3$.

The passage from the Lebesgue spaces to the Lorentz spaces estimates for those semigroups is made using the real interpolation method.

Proposition 2.2. Under the assumptions of Proposition 2.1,

$$
\left\|T_{u_{\infty}}(t) v_{0}\right\|_{L^{q, r}} \leq c t^{-\frac{3}{2}\left(\frac{1}{p}-\frac{1}{q}\right)}\left\|v_{0}\right\|_{L^{p, r}}
$$

for $t>0,1<p \leq q<\infty, 1 \leq r \leq \infty$, and

$$
\left\|\nabla T_{u_{\infty}}(t) v_{0}\right\|_{L^{q, r}} \leq c t^{-\frac{3}{2}\left(\frac{1}{p}-\frac{1}{q}\right)-\frac{1}{2}}\left\|v_{0}\right\|_{L^{p, r}}
$$

for $t>0,1<p \leq q \leq 3,1 \leq r \leq \infty$.

These are particular cases of the estimates recorded in [18]. The estimates for the Stokes semigroup with $u_{\infty}=0$ have been proved earlier in [20].

In the following, we also use the integral estimate

$$
\int_{0}^{\infty} t^{\frac{3}{2}\left(\frac{1}{p}-\frac{1}{q}\right)-\frac{1}{2}}\left\|\nabla T_{u_{\infty}}(t) v_{0}\right\|_{L^{q, 1}} d t \leq C\left\|v_{0}\right\|_{L^{p, 1}}
$$

which holds true under the assumptions of Proposition 2.1 and which is valid for all $v_{0} \in L_{\sigma}^{p, 1}(\Omega), 1<p \leq q \leq 3$, and a constant $C>0$. In the case of the Stokes semigroup, inequality (2.9) is due to Yamazaki [20, Cor. 2.3]. For the Oseen semigroup $T_{u_{\infty}}(t)$, the analogous result holds true which is a consequence of the real interpolation method applied to (2.8) and a reasoning in the proof of [20, Cor. 2.3].

We will also need some technical lemmata which we formulate below.

Lemma 2.1. Under the assumptions of Proposition 2.1, the following estimate is satisfied:

$$
\left\|\int_{0}^{t} T_{u_{\infty}}(t-s) \mathbb{P} \nabla G(s) d s\right\|_{L^{3, \infty}} \leq C \sup _{0 \leq s \leq t}\|G(s)\|_{L^{3 / 2, \infty}}
$$

for each function $G \in L^{\infty}\left((0, \infty) ; L^{3 / 2, \infty}(\Omega)\right)$, all $t>0$, and a constant $C>0$.

Proof. The proof of this lemma can be inferred from the reasoning contained in $[20,18]$; here, we reproduce it for the completeness of the exposition. Given a test function $\varphi \in$ $L_{\sigma}^{3 / 2,1}(\Omega)$ it follows from the Hölder inequality in Lorentz spaces that

$$
\begin{aligned}
\left|\left\langle\int_{0}^{t} T_{u_{\infty}}(t-s) \mathbb{P} \nabla G(s) d s, \varphi\right\rangle\right| & \leq \int_{0}^{t}\left|\left\langle G(s), \nabla T_{u_{\infty}}(t-s) \varphi\right\rangle\right| d s \\
& \leq \sup _{0 \leq s \leq t}\|G(s)\|_{L^{3 / 2, \infty}} \int_{0}^{t}\left\|\nabla T_{u_{\infty}}(t-s) \varphi\right\|_{L^{3,1}} d s .
\end{aligned}
$$

Now, the Yamazaki estimate (2.9) leads to the majorization of the second factor on the right hand side by

$$
\int_{0}^{t}\left\|\nabla T_{u_{\infty}}(s) \varphi\right\|_{L^{3,1}} d s \leq \int_{0}^{\infty}\left\|\nabla T_{u_{\infty}}(s) \varphi\right\|_{L^{3,1}} d s \leq C\|\varphi\|_{L^{3 / 2,1}} .
$$

The duality argument completes the proof. 
Lemma 2.2. Under the assumptions of Proposition 2.1, if $G \in \mathcal{C}_{w}\left([0, \infty) ; L^{3 / 2, \infty}(\Omega)\right)$ satisfies $\lim _{t \rightarrow \infty}\|G(t)\|_{L^{3 / 2, \infty}}=0$, then

$$
\lim _{t \rightarrow \infty}\left\|\int_{0}^{t} T(t-s) \mathbb{P} \nabla G(s) d s\right\|_{L^{3, \infty}}=0 .
$$

Proof. An analogous result in the case of the whole space $\Omega=\mathbb{R}^{3}$ can be found in [2]. We split the integral into the sum $\int_{0}^{t / 2} \ldots+\int_{t / 2}^{t} \ldots$, and proceeding as in the proof of Lemma 2.1 with $\varphi \in L_{\sigma}^{3 / 2,1}(\Omega)$ we estimate

$$
\begin{aligned}
\left|\left\langle\int_{0}^{t / 2} T(t-s) \mathbb{P} \nabla G(s) d s, \varphi\right\rangle\right| & \leq \int_{0}^{t / 2}\|G(s)\|_{L^{3 / 2, \infty}}\|\nabla T(t-s) \varphi\|_{L^{3,1}} d s \\
& \leq C \int_{0}^{t / 2}\|G(s)\|_{L^{3 / 2, \infty}}(t-s)^{-1}\|\varphi\|_{L^{3 / 2,1}} d s .
\end{aligned}
$$

Hence, by duality, we have

$$
\begin{aligned}
\left\|\int_{0}^{t / 2} T(t-s) \mathbb{P} \nabla G(s) d s\right\|_{L^{3 / 2, \infty}} & \leq C \int_{0}^{t / 2}(t-s)^{-1}\|G(s)\|_{L^{3 / 2, \infty}} d s \\
& \leq C \int_{0}^{1 / 2}(1-\tau)^{-1}\|G(t \tau)\|_{L^{3 / 2, \infty}} d \tau
\end{aligned}
$$

which tends to 0 as $t \rightarrow \infty$ by the Lebesgue dominated convergence theorem.

To bound the second integral term we apply Lemma 2.1 with the function $G$ restricted to the interval $[t / 2, t]$ and equal 0 otherwise. Hence, we obtain

$$
\left\|\int_{t / 2}^{t} T(t-s) \mathbb{P} \nabla G(s) d s\right\|_{L^{3, \infty}} \leq C \sup _{t / 2 \leq s \leq t}\|G(s)\|_{L^{3 / 2, \infty}} \rightarrow 0
$$

as $t \rightarrow \infty$, by the assumption on $G$.

3. Existence and stability of solutions for the nonlinear problem. In the remainder of the paper we assume that the external forces are of potential type, that is $f$ is derived from a tensor function $F=\left(F_{j k}(x)\right)_{j, k=1}^{3}$, so that

$$
\left(f_{j}(x)\right)_{j=1}^{3}=\nabla \cdot F(x)=\left(\sum_{k=1}^{3} \frac{\partial F_{j k}}{\partial x_{k}}(x)\right)_{j=1}^{3} .
$$

The forces $F$ may also depend on time if some continuity assumptions are made.

Definition 3.1. A function $u \in \mathcal{X}=\mathcal{C}_{w}\left([0, \infty) ; L_{\sigma}^{3, \infty}(\Omega)\right)$ is said to be a mild solution of the Navier-Stokes system (1.1)-(1.5) if

$$
u(t)=T_{u_{\infty}}(t) u_{0}-\int_{0}^{t} T_{u_{\infty}}(t-s)[\mathbb{P} \nabla(u \otimes u)(s)+\mathbb{P} \nabla \cdot F(s)] d s .
$$

The above integral is understood in the weak sense, i.e. the identity

$$
\langle u(t), \varphi\rangle=\left\langle u_{0}, T_{u_{\infty}}(t) \varphi\right\rangle+\sum_{j, k=1}^{3} \int_{0}^{t}\left\langle u_{j}(s) u_{k}(s)-F_{j k}(s), \frac{\partial}{\partial x_{j}}\left(T_{-u_{\infty}}(t-s) \varphi\right)_{k}\right\rangle d s
$$

holds for every test function $\varphi \in L_{\sigma}^{3 / 2,1}(\Omega)$ and all $t>0$. 
Note that in the case $\Omega \neq \mathbb{R}^{3}$, the operators $\nabla$ and $T_{u_{\infty}}(t)$ do not commute. Thus, the estimates on the decay of $\nabla T_{u_{\infty}}(t)$ could not be applied directly to the integral formulation (3.1), so the weak formulation is more suitable.

In the three-dimensional domains, the Lorentz class $L_{\sigma}^{3, \infty}(\Omega)$ is a natural generalization of the class of physically reasonable solutions satisfying the asymptotic relation $u(x) \sim 1 /|x|$ as $|x| \rightarrow \infty$, see [5]. The reason for considering the space of weakly continuous vector valued functions $\mathcal{C}_{w}\left([0, \infty) ; L_{\sigma}^{3, \infty}(\Omega)\right)$ is that the Stokes and the Oseen semigroups on $L_{\sigma}^{3, \infty}(\Omega)$ (like the heat semigroup $e^{t \Delta}$ on the Lorentz space $L^{3, \infty}\left(\mathbb{R}^{3}\right)$, cf. e.g. [2]) are not strongly continuous at $t=0$. Hence, instead of the space of strongly continuous functions $C\left([0, \infty) ; L_{\sigma}^{3, \infty}(\Omega)\right)$, we choose the space $\mathcal{C}_{w}\left([0, \infty) ; L_{\sigma}^{3, \infty}(\Omega)\right)$ of bounded vector valued functions which are strongly continuous in $(0, \infty)$, and weakly continuous as $t \searrow 0$.

For three-dimensional exterior domains, the best spatial decay expected is, in general, $u(t) \in L^{3, \infty}(\Omega)$. Thus, the expression in the integral formulation (3.1) might not be Bochner integrable. The weak formulation circumvents this difficulty. For an explicit example of such a non-Bochner integrable expression in (3.1) related to forward self-similar solutions of the Navier-Stokes equations in $\mathbb{R}^{3}$, we refer the reader to [20, Remark 1.2], see also [2].

We begin with the case $u_{\infty}=0$ which is a bit simpler. The basic estimate which leads to a proof of existence of solutions, as well as to a proof of stability, is the boundedness of the quadratic term in the Navier-Stokes equations in the space

$$
\mathcal{X}=\mathcal{C}_{w}\left([0, \infty) ; L_{\sigma}^{3, \infty}(\Omega)\right)
$$

chosen in our functional framework.

Proposition 3.1. The bilinear form $B$ defined by

$$
B(u, v)(t)=-\int_{0}^{t} T(t-s) \mathbb{P} \nabla \cdot(u \otimes v)(s) d s
$$

is bounded on the space $\mathcal{X}$, i.e. $\|B(u, v)\|_{\mathcal{X}} \leq \eta\|u\|_{\mathcal{X}}\|v\|_{\mathcal{X}}$ for all $u, v \in \mathcal{X}$ and a constant $\eta>0$.

The proof of Proposition 3.1 is contained in [20, Th. 3.1]. A different proof by Y. Meyer in the case of $\Omega=\mathbb{R}^{3}$ can be found in [15, Ch. 18].

Theorem 3.1. Assume that $u_{0} \in L_{\sigma}^{3, \infty}(\Omega)$ and $F \in L^{3 / 2, \infty}(\Omega)$ satisfy $\left\|u_{0}\right\|_{L^{3, \infty}}+$ $\|F\|_{L^{3 / 2, \infty}}<\varepsilon$ for some $0<\varepsilon<1 /(4 \eta)$, where $\eta$ is defined in the Proposition 3.1. There exists a global in time solution of (1.1)-(1.5) in the space $\mathcal{X}=\mathcal{C}_{w}\left([0, \infty) ; L_{\sigma}^{3, \infty}(\Omega)\right)$. This is the unique solution satisfying the condition $\|u\|_{\mathcal{X}} \leq 2 \varepsilon$. Moreover, this solution depends continuously on the initial data and the external forces.

Proof. This is an application of the estimate of the bilinear form (3.2) from Proposition 3.1 and the Banach fixed point theorem, see e.g. [20, 17, 2].

REMARK 3.1. It is easy to modify the construction of solutions in Theorem 3.1 to the case of nonautonomous forces $F \in \mathcal{C}_{w}\left([0, \infty) ; L^{3 / 2, \infty}(\Omega)\right)$ (cf. [20]). 
In the case of $u_{\infty} \neq 0$, first, the existence of a stationary solution $w$ is established, and then the evolution equation for $u-w$ is solved. The precise statements are given below.

Theorem 3.2 ([19, Theorems 1.1 and 1.2]). There exists $\varepsilon_{1}>0$ such that if $F \in$ $L^{3 / 2, \infty}(\Omega)$ and $u_{\infty} \in \mathbb{R}^{3}$ satisfy $\sum_{j, k=1}^{3}\left\|F_{j k}\right\|_{L^{3 / 2, \infty}}+\left|u_{\infty}\right| \leq \varepsilon_{1}$, then the stationary problem

$$
\begin{aligned}
-\Delta w+\left(u_{\infty} \cdot \nabla\right) w+(w \cdot \nabla) w+\nabla \pi & =\nabla \cdot F, \\
\nabla \cdot w & =0, \\
w_{\mid \partial \Omega} & =-u_{\infty}, \\
\lim _{|x| \rightarrow \infty} w(x) & =0,
\end{aligned}
$$

admits a solution $w \in L_{\sigma}^{3, \infty}(\Omega)$ with $\nabla w \in L^{3 / 2, \infty}(\Omega)$, and it satisfies the estimate

$$
\|\nabla w\|_{L^{3 / 2, \infty}}+\|w\|_{L^{3, \infty}}+\|\pi\|_{L^{3 / 2, \infty}} \leq C \varepsilon_{1}
$$

with a constant $C$ independent of $F, w, \pi, \varepsilon_{1}$ and $u_{\infty}$. This solution is unique in the class of small $L_{\sigma}^{3, \infty}(\Omega)$ functions.

REMARK 3.2. In general, the solution $w$ of the stationary problem (1.1)-(1.5) with $u_{\infty}=$ 0 (even if it is small enough) does not belong to the space $L^{3}(\Omega)$, in contrast to the case when $u_{\infty} \neq 0$ and $w-u_{\infty} \in L^{3}(\Omega)$. The recent paper [19] deals with the space asymptotics of the stationary solutions of the Navier-Stokes equations when $u_{\infty} \rightarrow 0$.

In the evolution case, the solution of the perturbed problem (obtained using again a contraction argument) is described in the following result based on [18, Th. 5.1].

TheOREM 3.3. Assume that $w=w(x)$ is the stationary solution constructed in Theorem 3.2. There exists $\varepsilon_{2}>0$ such that if $z_{0} \in L_{\sigma}^{3, \infty}(\Omega)$ satisfy $\left\|z_{0}\right\|_{L^{3, \infty}}+\|\Phi\|_{L^{3 / 2, \infty}} \leq \varepsilon_{2}$, then the problem

$$
\begin{aligned}
z_{t}-\Delta z+(w \cdot \nabla) z+(z \cdot \nabla) w+(z \cdot \nabla z) z+\nabla \varrho & =\nabla \cdot \Phi, \\
\nabla \cdot z & =0, \\
z_{\mid \partial \Omega} & =0, \\
z(x, 0) & =z_{0}(x), \\
\lim _{|x| \rightarrow \infty} z(x, t) & =0,
\end{aligned}
$$

with $z_{0}=u_{0}-u_{\infty}-w$, admits a unique solution $z$ in $\mathcal{C}_{w}\left([0, \infty) ; L_{\sigma}^{3, \infty}(\Omega)\right)$ satisfying the estimate $\sup _{t>0}\|z(t)\|_{L^{3, \infty}} \leq C \varepsilon_{2}$ with a constant $C$ independent of $u_{\infty}$ and $u_{0}$.

The proof of Theorem 3.3 is an obvious modification of the corresponding result contained in [18] where $\Phi=0$.

Now we formulate our main results on the asymptotic stability of solutions in the weak Lebesgue space $L^{3, \infty}(\Omega)$. We begin with the case $u_{\infty}=0$.

TheOREm 3.4. Assume that $u$ and $\tilde{u}$ are two solutions of the problem (1.1)-(1.5) with $u_{\infty}=0$ constructed in Theorem 3.1 and corresponding to the initial conditions $u_{0}, \widetilde{u}_{0} \in$ 
$L_{\sigma}^{3, \infty}(\Omega)$ and the external forces $F, \widetilde{F} \in \mathcal{C}_{w}\left([0, \infty) ; L^{3 / 2, \infty}(\Omega)\right)$, respectively. Suppose that

$$
\lim _{t \rightarrow \infty}\left\|T(t)\left(u_{0}-\widetilde{u}_{0}\right)\right\|_{L^{3, \infty}}=0 \text { and } \lim _{t \rightarrow \infty}\|F(t)-\widetilde{F}(t)\|_{L^{3 / 2, \infty}}=0 .
$$

Then

$$
\lim _{t \rightarrow \infty}\|u(t)-\widetilde{u}(t)\|_{L^{3, \infty}}=0 .
$$

The above result means that if the difference of the solutions of the Stokes problem issued from $u_{0}$ and $\widetilde{u}_{0}$ becomes negligible as $t \rightarrow \infty$ (this holds if, e.g., the difference of the initial data $u_{0}$ and $\widetilde{u}_{0}$ is not too singular), and if $F(t)$ and $\widetilde{F}(t)$ have the same large time asymptotics, the solutions of the full nonlinear Navier-Stokes problem (1.1)-(1.5) behave similarly for large times. It can be interpreted as a kind of asymptotic stability when the choice of $\widetilde{u}_{0}$ is restricted to the initial data in a neighborhood of $u_{0}$ satisfying additionally (3.12). It is easy to verify that the first condition in (3.12) is satisfied if, e.g., $u_{0}-\widetilde{u}_{0} \in L^{p}(\Omega) \cap L_{\sigma}^{3, \infty}(\Omega)$ with some $1 \leq p<3$.

In the case $u_{\infty} \neq 0$ we have a slightly different statement but the proof will be practically the same.

THEOREM 3.5. Let $z$ be the solution of the perturbed problem constructed in Theorem 3.3 corresponding to the initial data $z_{0} \in L_{\sigma}^{3, \infty}(\Omega)$ such that

$$
\lim _{t \rightarrow \infty}\left\|T_{u_{\infty}}(t) z_{0}\right\|_{L^{3, \infty}}=0
$$

Then $\lim _{t \rightarrow \infty}\|z(t)\|_{L^{3, \infty}}=0$ is satisfied.

Recall that $z=u-w$ is the difference of the solution of the evolution problem (1.1)(1.5) and the steady state solution of (3.3)-(3.6) corresponding to the same asymptotic velocity $u_{\infty}$. Thus, unlike the situation in the preceding Theorem 3.4 for $u_{\infty}=0$, we consider here the asymptotic stability of stationary solutions, only.

With Lemmata 2.1-2.2, we are ready to begin the proofs of Theorems 3.4 and 3.5.

Proof of Theorem 3.4. We assume that $u_{\infty}=0$. For $u_{\infty} \neq 0$, the solutions $u, \tilde{u}$ should $\operatorname{read} z, w$ and the Duhamel formula (the counterpart of (3.1)) used in this case is

$$
z(t)=T_{u_{\infty}}(t) z_{0}-\int_{0}^{t} T_{u_{\infty}}(t-s) \mathbb{P}(\nabla \cdot(w \otimes z(s)+z(s) \otimes w+z(s) \otimes z(s))) d s,
$$

(see also [18, p. 163]).

Recall first that, by Theorem 3.1 , we have

$$
\sup _{t \geq 0}\|u(t)\|_{L^{3, \infty}} \leq 2 \varepsilon<\frac{1}{2 \eta} \quad \text { and } \sup _{t \geq 0}\|\widetilde{u}(t)\|_{L^{3, \infty}} \leq 2 \varepsilon<\frac{1}{2 \eta} .
$$

Subtracting the integral equation (3.1) for $\widetilde{u}$ from the analogous expression for $u$ we get

$$
\begin{aligned}
\|u(t)-\widetilde{u}(t)\|_{L^{3, \infty}} \leq & \left\|T(t)\left(u_{0}-\widetilde{u}_{0}\right)\right\|_{L^{3, \infty}} \\
& +\left\|\int_{0}^{t} T(t-s) \mathbb{P} \nabla \cdot((u-\widetilde{u}) \otimes u+\widetilde{u} \otimes(u-\widetilde{u}))(s) d s\right\|_{L^{3, \infty}} \\
& +\left\|\int_{0}^{t} T(t-s) \mathbb{P} \nabla \cdot(F(s)-\widetilde{F}(s)) d s\right\|_{L^{3, \infty}}
\end{aligned}
$$




$$
\begin{aligned}
\leq & \left\|\int_{0}^{\delta t} T(t-s) \mathbb{P} \nabla \cdot((u-\widetilde{u}) \otimes u+\widetilde{u} \otimes(u-\widetilde{u}))(s) d s\right\|_{L^{3, \infty}} \\
& +\left\|\int_{\delta t}^{t} T(t-s) \mathbb{P} \nabla \cdot((u-\widetilde{u}) \otimes u+\widetilde{u} \otimes(u-\widetilde{u}))(s) d s\right\|_{L^{3, \infty}}+g(t),
\end{aligned}
$$

where the function $g=g(t)$ is the sum of the first and the third terms in (3.15), and the number $0<\delta<1$ will be determined later.

The norm of the integral $\int_{0}^{\delta t} \ldots$ above is estimated using the Yamazaki estimates (2.7)-(2.8) combined with the weak Hölder inequality and with the bound on solutions in Theorem 3.1 by the following quantity:

$$
\begin{aligned}
& C \int_{0}^{\delta t}(t-s)^{-1}\|((u-\widetilde{u}) \otimes u+\widetilde{u} \otimes(u-\widetilde{u}))(s)\|_{L^{3 / 2, \infty}} d s \\
& \quad \leq C \int_{0}^{\delta t}(t-s)^{-1}\left(\|u(s)\|_{L^{3, \infty}}+\|\widetilde{u}(s)\|_{L^{3, \infty}}\right)\left(\|u(s)\|_{L^{3, \infty}}+\|\widetilde{u}(s)\|_{L^{3, \infty}}\right) d s \\
& \quad \leq C(4 \varepsilon)^{2} \int_{0}^{\delta t}(t-s)^{-1} d s=C(4 \varepsilon)^{2} \log \left(\frac{1}{1-\delta}\right) .
\end{aligned}
$$

The norm of the $\int_{\delta t}^{t} \ldots$ integral is bounded from above by using Lemma 2.1 with $G=(u-\widetilde{u}) \otimes u+\widetilde{u} \otimes(u-\widetilde{u})$ on the interval $[\delta t, t]$ and 0 otherwise, combined again with the weak Hölder inequality, by

$$
\begin{aligned}
\eta\left(\sup _{\delta t \leq s \leq t}\|u(s)\|_{L^{3, \infty}}+\sup _{\delta t \leq s \leq t}\|\widetilde{u}(s)\|_{L^{3, \infty}}\right) \sup _{\delta t \leq s \leq t}\|u(s)-\widetilde{u}(s)\|_{L^{3, \infty}} \\
\leq 4 \varepsilon \eta \sup _{\delta t \leq s \leq t}\|u(s)-\widetilde{u}(s)\|_{L^{3, \infty}} .
\end{aligned}
$$

Putting $A=\limsup \sup _{t \rightarrow \infty}\|u(t)-\widetilde{u}(t)\|_{L^{3, \infty}}=\lim _{k \in \mathbb{N}, k \rightarrow \infty} \sup _{t>k}\|u(t)-\widetilde{u}(t)\|_{L^{3, \infty}}$, we see that $0 \leq A<\infty$ since $u, \widetilde{u} \in L^{\infty}\left((0, \infty) ; L_{\sigma}^{3, \infty}(\Omega)\right)$. We claim that $A=0$. Since $\sup _{t \geq k} \sup _{\delta t \leq s \leq t}\|u(s)-\widetilde{u}(s)\|_{L^{3, \infty}} \leq \sup _{\delta k \leq s<\infty}\|u(s)-\widetilde{u}(s)\|_{L^{3, \infty}}$, we have

$$
\limsup _{t \rightarrow \infty}\left(\sup _{\delta t \leq s \leq t}\|u(s)-\widetilde{u}(s)\|_{L^{3, \infty}}\right) \leq A .
$$

Moreover, the function $g$ is, by the assumptions and Lemma 2.2, bounded and converges to 0 as $t \rightarrow \infty$. Finally, computing the $\limsup _{t \rightarrow \infty}$ of both sides of (3.15) and using (3.16), (3.17), we arrive at

$$
A \leq C(4 \varepsilon)^{2} \log \left(\frac{1}{1-\delta}\right)+4 \varepsilon \eta A .
$$

Since $4 \varepsilon \eta<1$ as was assumed in Theorem 3.1, we get

$$
A \leq(1-4 \varepsilon \eta)^{-1} C(4 \varepsilon)^{2} \log \left(\frac{1}{1-\delta}\right),
$$

which can be made arbitrarily small by taking a suitable $0<\delta<1$. Therefore $A=0$, and this finishes the proof.

4. Regularity properties of solutions. The solutions $u$ and $z$ constructed in Theorems 3.1, 3.3 enjoy some a posteriori regularization effect as well as a better decay for 
$t \rightarrow \infty$. Constructed a priori in $L_{\sigma}^{3, \infty}(\Omega)$, they belong to $L^{p, \infty}(\Omega)$ with any $3<p<\infty$, and satisfy the estimates

$$
\|u(t)-\widetilde{u}(t)\|_{L^{p, \infty}} \leq C t^{-\frac{3}{2}\left(\frac{1}{3}-\frac{1}{p}\right)} \quad \text { and } \quad\|z(t)\|_{L^{p, \infty}} \leq C t^{-\frac{3}{2}\left(\frac{1}{3}-\frac{1}{p}\right)},
$$

respectively (for the proof, see $[20,19]$ ). As the complements of Theorems 3.4 and 3.5, we are able to improve these two inequalities.

First, however, we need some preliminary results. For a function

$$
H \in L_{\mathrm{loc}}^{\infty}\left((0, \infty) ; L^{3 p /(p+3), \infty}(\Omega)\right) \quad \text { with } p \geq 3
$$

we define an auxiliary norm

$$
\|H\|_{p, \infty} \equiv \sup _{t>0} t^{\frac{3}{2}\left(\frac{1}{3}-\frac{1}{p}\right)}\|H(t)\|_{L^{3 p /(p+3), \infty}} .
$$

It is obvious that $\|H\|_{3, \infty}=\sup _{t>0}\|H(t)\|_{L^{3 / 2, \infty}}$.

Using this new notation we have the following generalizations of Lemmata 2.1 and 2.2.

Lemma 4.1. For each function $H \in L_{\text {loc }}^{\infty}\left((0, \infty) ; L^{3 p /(p+3), \infty}(\Omega)\right), p \geq 3$, the following estimate holds:

$$
t^{\frac{3}{2}\left(\frac{1}{3}-\frac{1}{p}\right)}\left\|\int_{0}^{t} T_{u_{\infty}}(t-s) \mathbb{P} \nabla H(s) d s\right\|_{L^{p, \infty}} \leq C\|H\|_{p, \infty}
$$

with a constant $0<C<\infty$.

Proof. The idea is essentially that of [18, proof of Th. 5.1], see also [2, Lemma 7.3]. For any test function $\varphi \in L_{\sigma}^{q, 1}(\Omega)$ and $1 / p+1 / q=1$, we are going to estimate the following quantity:

$$
\begin{aligned}
&\left|\left\langle t^{\frac{3}{2}\left(\frac{1}{3}-\frac{1}{p}\right)} \int_{0}^{t} T_{u_{\infty}}(t-s) \mathbb{P} \nabla H(s) d s, \varphi\right\rangle\right| \\
&=t^{\frac{3}{2}\left(\frac{1}{3}-\frac{1}{p}\right)}\left|\int_{0}^{t}\left\langle H(s), \nabla T_{-u_{\infty}}(t-s) \varphi\right\rangle d s\right| .
\end{aligned}
$$

Splitting the integral over $[0, t]$ into $\int_{0}^{t / 2} \ldots+\int_{t / 2}^{t} \ldots$, we bound the first of them by

$$
C\left(\sup _{0 \leq s \leq t} s^{\frac{3}{2}\left(\frac{1}{3}-\frac{1}{p}\right)}\|H(s)\|_{L^{\mu, \infty}}\right)\left(t^{\frac{3}{2}\left(\frac{1}{3}-\frac{1}{p}\right)} \int_{0}^{t / 2}(t-s)^{-\frac{3}{2}\left(\frac{1}{q}-\frac{1}{\nu}\right)-\frac{1}{2}} s^{-\frac{3}{2}\left(\frac{1}{3}-\frac{1}{p}\right)} d s\right)\|\varphi\|_{L^{q, 1}}
$$

with $1 / q-1 / \nu=1 / \mu-1 / p=1 / 3$, and then by

$$
C\left(\sup _{0 \leq s \leq t} s^{\frac{3}{2}\left(\frac{1}{3}-\frac{1}{p}\right)}\|H(s)\|_{L^{\mu, \infty}}\right)\|\varphi\|_{L^{q, 1}} .
$$

The remaining term is bounded by

$$
\begin{aligned}
C t^{\frac{3}{2}\left(\frac{1}{3}-\frac{1}{p}\right)}\left(\frac{t}{2}\right)^{-\frac{3}{2}\left(\frac{1}{3}-\frac{1}{p}\right)} \int_{t / 2}^{t} \| \nabla T_{-u_{\infty}}( & t-s) \varphi \|_{L^{\nu, 1}} d s \\
& \leq C \int_{0}^{\infty}\left\|\nabla T_{-u_{\infty}}(s) \varphi\right\|_{L^{\nu, 1}} d s \leq C\|\varphi\|_{L^{q, 1}}
\end{aligned}
$$

after an application of the estimate (2.9).

The duality argument completes the proof. 
LEMMA 4.2. Under the assumptions of Lemma 4.1, if moreover

$$
\lim _{t \rightarrow \infty} t^{\frac{3}{2}\left(\frac{1}{3}-\frac{1}{p}\right)}\|H(t)\|_{L^{3 p /(p+3), \infty}}=0
$$

and if either $H \in \mathcal{C}_{w}\left([0, \infty) ; L^{p, \infty}(\Omega)\right)$ or $\sup _{\delta t \leq s \leq t} s^{\frac{3}{2}\left(\frac{1}{3}-\frac{1}{p}\right)}\|H(s)\|_{L^{3 p /(p+3), \infty}} \rightarrow 0$ as $t \rightarrow \infty$, then

$$
\lim _{t \rightarrow \infty} t^{\frac{3}{2}\left(\frac{1}{3}-\frac{1}{p}\right)}\left\|\int_{0}^{t} T_{u_{\infty}}(t-s) \mathbb{P} \nabla H(s) d s\right\|_{L^{p, \infty}}=0 .
$$

Proof. We split the integral into $\int_{0}^{\delta t} \ldots+\int_{\delta t}^{t} \ldots$, and estimate the two terms separately. First, it follows from the semigroup estimates contained in Proposition 2.2 that

$$
\begin{aligned}
\left\|\int_{0}^{\delta t} T_{u_{\infty}}(t-s) \mathbb{P} \nabla H(s) d s\right\|_{L^{p, \infty}} & \leq C \int_{0}^{\delta t}(t-s)^{-\frac{3}{2}\left(\frac{1}{3}-\frac{1}{p}\right)-1} d s \cdot \sup _{s>0}\|H(s)\|_{L^{3 / 2, \infty}} \\
& =C\left(1-(1-\delta)^{-\frac{3}{2}\left(\frac{1}{3}-\frac{1}{p}\right)}\right) t^{-\frac{3}{2}\left(\frac{1}{3}-\frac{1}{p}\right)}=o(1) t^{-\frac{3}{2}\left(\frac{1}{3}-\frac{1}{p}\right)}
\end{aligned}
$$

as $\delta \searrow 0$. Second, applying Lemma 4.1 directly, we arrive at

$$
\left\|\int_{\delta t}^{t} T_{u_{\infty}}(t-s) \mathbb{P} \nabla H(s) d s\right\|_{L^{p, \infty}} \leq C t^{-\frac{3}{2}\left(\frac{1}{3}-\frac{1}{p}\right)} \sup _{\delta t \leq s \leq t} s^{\frac{3}{2}\left(\frac{1}{3}-\frac{1}{p}\right)}\|H(s)\|_{L^{3 p /(p+3), \infty},}
$$

which is $o\left(t^{-\frac{3}{2}\left(\frac{1}{3}-\frac{1}{p}\right)}\right)$ at $t \rightarrow \infty$ by the assumption on the function $H$.

Now, we are ready to improve inequalities recalled in (4.1).

THEOREM 4.1. Under the assumptions of Theorem 3.4, if additionally for some $3<p<$ $\infty$,

$$
\sup _{t>0} t^{\frac{3}{2}\left(\frac{1}{3}-\frac{1}{p}\right)}\|F(t)-\widetilde{F}(t)\|_{L^{3 p /(p+3), \infty}}<\infty
$$

then

$$
\lim _{t \rightarrow \infty} t^{\frac{3}{2}\left(\frac{1}{3}-\frac{1}{p}\right)}\|u(t)-\widetilde{u}(t)\|_{L^{p, \infty}}=0 .
$$

Proof. It follows from [20, Th. 1.3] that solutions $u$ and $\widetilde{u}$ in Theorem 4.1 satisfy $u(t)-$ $\widetilde{u}(t) \in L^{p, \infty}(\Omega)$ for each $3<p<\infty$. Moreover,

$$
\sup _{t>0} t^{\frac{3}{2}\left(\frac{1}{3}-\frac{1}{p}\right)}\|u(t)-\widetilde{u}(t)\|_{L^{p, \infty}}<\infty
$$

In order to prove that the above quantity tends to 0 as $t \rightarrow \infty$, we decompose the difference of the solutions as

$$
\begin{aligned}
u(t)-\widetilde{u}(t)= & T(t)\left(u_{0}-\widetilde{u}_{0}\right)+\int_{0}^{t} T(t-s) \mathbb{P} \nabla \cdot(u \otimes(u-\widetilde{u}))(s) d s \\
& +\int_{0}^{t} T(t-s) \mathbb{P} \nabla \cdot((u-\widetilde{u}) \otimes \widetilde{u})(s) d s+\int_{0}^{t} T(t-s) \mathbb{P} \nabla \cdot(F(s)-\widetilde{F}(s)) d s .
\end{aligned}
$$

Now we estimate the $L^{p, \infty}(\Omega)$ norms of each of the above terms. First we have

$$
\left\|T(t)\left(u_{0}-\widetilde{u}_{0}\right)\right\|_{L^{p, \infty}} \leq C\left(\frac{t}{2}\right)^{-\frac{3}{2}\left(\frac{1}{3}-\frac{1}{p}\right)}\left\|T\left(\frac{t}{2}\right)\left(u_{0}-\widetilde{u}_{0}\right)\right\|_{L^{3, \infty}}
$$

which is $\left.o^{-\frac{3}{2}\left(\frac{1}{3}-\frac{1}{p}\right)}\right)$ as $t \rightarrow \infty$ by the assumption on the behavior of the Stokes semigroup applied to $u_{0}-\widetilde{u}_{0}$. 
The second and the third integrals are estimated in a completely analogous way using Lemma 4.1 with $H=u \otimes(u-\widetilde{u})$ and $H=(u-\widetilde{u}) \otimes \widetilde{u}$, resp. Note that here $\|H(s)\|_{L^{3 p /(p+3), \infty}} \leq C\|u(s)\|_{L^{3, \infty}}\|u(s)-\widetilde{u}(s)\|_{L^{p, \infty}}$ by the weak Hölder inequality.

The last fourth term is estimated directly from Lemma 4.2.

Essentially the same arguments as in the proof of Theorem 4.1 apply to the problem (3.7)-(3.11) leading to the following result.

THEOREM 4.2. Under the assumptions of Theorem 3.5, if $\lim _{\rightarrow \infty}\left\|T_{u_{\infty}}(t) z_{0}\right\|_{L^{3, \infty}}=0$, then $\lim _{t \rightarrow \infty} t^{\frac{3}{2}\left(\frac{1}{3}-\frac{1}{p}\right)}\|z(t)\|_{L^{p, \infty}}=0$.

Acknowledgements. The preparation of this paper was partially supported by the KBN grant 2/P03A/002/24, the POLONIUM project ÉGIDE-KBN 2002-2003, and the EU contract HYKE No. HPRN-CT-2002-00282. A part of this paper was written when the first named author was a visiting professor at Université de Marne-la-Vallée.

\section{References}

[1] W. Borchers and T. Miyakawa, On stability of exterior stationary Navier-Stokes flows, Acta Math. 174 (1995), 311-382.

[2] M. Cannone and G. Karch, About the regularized Navier-Stokes equations, J. Mathematical Fluid Mechanics, in print.

[3] N. Depauw, Solutions des équations de Navier-Stokes incompressibles dans un domaine extérieur, Rev. Mat. Iberoamericana 17 (2001), 21-68.

[4] R. Finn, An energy theorem for viscous fluid motions, Arch. Rational Mech. Anal. 6 (1960), 371-381.

[5] R. Finn, On exterior stationary problem for the Navier-Stokes equations and associated perturbation problems, Arch. Rational Mech. Anal. 19 (1965), 363-406.

[6] R. Finn, Stationary solutions of the Navier-Stokes equations, in: Proc. Symp. Appl. Math. 17, Amer. Math. Soc., Providence, 1965, 121-153.

[7] G. P. Galdi, An Introduction to the Mathematical Theory of the Navier-Stokes Equations, Vol. I and II, Springer, New York, 1994.

[8] G. P. Galdi, J. G. Heywood and Y. Shibata, On the global existence and convergence to steady state of Navier-Stokes flow past an obstacle that is started from the rest, Arch. Rational Mech. Anal. 138 (1997), 307-318.

[9] J. Heywood, On stationary solutions of the Navier-Stokes equations as limits of nonstationary solutions, Arch. Rational Mech. Anal. 37 (1970), 48-60.

[10] T. Kato, Strong $L^{p}$ solutions of the Navier-Stokes equations in $\mathbb{R}^{m}$ with applications to weak solutions, Math. Z. 187 (1984), 471-480.

[11] T. Kobayashi and Y. Shibata, On the Oseen equation in the three-dimensional exterior domains, Math. Ann. 310 (1998), 1-45.

[12] H. Kozono and M. Yamazaki, Exterior problem for the stationary Navier-Stokes equations in the Lorentz spaces, Math. Ann. 310 (1998), 279-305.

[13] H. Kozono and M. Yamazaki, On a large class of stable solutions to the Navier-Stokes equations in exterior domains, Math. Z. 228 (1998), 751-785. 
[14] J. Leray, Étude de diverses équations intégrales non-linéaires et de quelques problèmes que pose l'hydrodynamique, J. Math. Pures et Appl. 12 (1933), 1-82.

[15] Y. Meyer, Wavelets, paraproducts and Navier-Stokes equations, in: Current Developments in Mathematics, R. Bott et al. (ed.), International Press, Cambridge, MA, 1999, 105-212.

[16] C. W. Oseen, Neueren Methoden und Ergebnisse in der Hydrodynamik, Akademische Verlaggesellschaft, Leipzig, 1927.

[17] Y. Shibata, On an exterior initial boundary value problem for Navier-Stokes equations, Quart. Appl. Math. 57 (1999), 117-155.

[18] Y. Shibata, On a stability theorem of the Navier-Stokes equations in a three dimensional exterior domain, in: Tosio Kato's Method and Principle for Evolution Equations in Mathematical Physics, H. Fujita, S. T. Kuroda and H. Okamoto (eds.), Yurinsha, Tokyo, 2002, 146-172 (appeared also in Sūrikaisekikenkyūsho Kōkyūroku 1234 (2001)).

[19] Y. Shibata and M. Yamazaki, Uniform estimates in the velocity at infinity for stationary solutions to the Navier-Stokes exterior problem, preprint, $60 \mathrm{pp}$.

[20] M. Yamazaki, The Navier-Stokes equations in the weak- $L^{n}$ space with time-dependent external force, Math. Ann. 317 (2000), 635-675. 